OPEN ACCESS

Edited by:

Luís Jaime Mota,

NOVA School of Science and

Technology, Portugal

Reviewed by:

Daniel Alford Powell,

University of Arizona, United States

*Correspondence:

Simone Filardo

simone.filardo@uniroma1.it

${ }^{t}$ These authors have contributed equally to this work and share first authorship

Specialty section:

This article was submitted to Molecular Bacterial Pathogenesis,

a section of the journal

Frontiers in Cellular and

Infection Microbiology

Received: 21 December 2021 Accepted: 11 January 2022

Published: 31 January 2022

Citation:

Filardo S, Di Pietro M, Diaco F and Sessa R (2022) In Vitro Modelling of Chlamydia trachomatis Infection in the Etiopathogenesis of Male Infertility and Reactive Arthritis. Front. Cell. Infect. Microbiol. 12:840802.

doi: 10.3389/fcimb.2022.840802

\section{In Vitro Modelling of Chlamydia trachomatis Infection in the Etiopathogenesis of Male Infertility and Reactive Arthritis}

\author{
Simone Filardo ${ }^{*+}$, Marisa Di Pietro ${ }^{\dagger}$, Fabiana Diaco and Rosa Sessa \\ Department of Public Health and Infectious Diseases, Section of Microbiology, University of Rome "Sapienza", Rome, Italy
}

Chlamydia trachomatis is an obligate, intracellular bacterium responsible for a range of diseases of public health importance, since $C$. trachomatis infection is often asymptomatic and, hence, untreated, leading to chronic complications, including prostatitis, infertility, and reactive arthritis. The ample spectrum of diseases caused by C. trachomatis infection is reflected in its ability to infect and multiply within a wide range of different cell types. Cervical epithelial cells, to date, have been the most studied cellular infection model, highlighting the peculiar features of the host-cell inflammatory and immune responses to the infection. Herein, we provide the up-to-date evidence on the interaction between C. trachomatis and human prostate epithelial, Sertoli and synovial cells.

Keywords: Chlamydia trachomatis, obligate intracellular bacteria, human prostate cells, human Sertoli cells, human synovial cells

\section{INTRODUCTION}

Chlamydia trachomatis, obligate, intracellular bacterium responsible for a range of diseases of public health importance, is the leading cause of sexually transmitted bacterial infection worldwide, with estimates of more than 130 million new cases each year (Rowley et al., 2019). In women, the most common clinical manifestations are cervicitis and urethritis, whereas in men these are urethritis and epididymitis, although in the majority of cases $C$. trachomatis genital infection is asymptomatic and, hence, untreated, leading to chronic complications, including prostatitis, infertility, and reactive arthritis (ReA) (O'Connell and Ferone, 2016; Di Pietro et al., 2019).

Prostatitis, one of the most common urologic problems for men younger than 50 years, and risk factor for infertility (Khan et al., 2017), has a prevalence of approximately $8 \%$ to $16 \%$, and around $5 \%$ to $10 \%$ of all cases have a bacterial origin, with C. trachomatis involved in up to $27 \%$ of all bacterial infections of the prostate (Ostaszewska et al., 1998; Badalyan et al., 2003; Krieger et al., 2008; Ouzounova-Raykova et al., 2010; Trinchieri et al., 2021). Nevertheless, chronic bacterial prostatitis is frequently underestimated because urinary tract infections often remain undocumented and thus neglected (Ouzounova-Raykova et al., 2010). Male infertility also remains a neglected area in sexual and reproductive health although it has a significant impact on public health worldwide, since approximately $15-20 \%$ of reproductive age couples are infertile in industrialized countries, and, in $30 \%$ of all cases, fertility problems are solely due to the male partner, with around 15\% of idiopathic cases attributed to infectious causes (Henkel et al., 2021; Thoma et al., 2021). Similarly, 
ReA is a frequently misdiagnosed condition, due to difficulties in relating past infections with compromised joint functions. It is estimated that approximately $4-8 \%$ of patients will develop ReA one to six weeks after a urogenital C. trachomatis infection, and in 30\% of all cases, ReA persists for years, leading, eventually, to joint deformities and ankylosis (Zeidler and Hudson, 2016; Di Pietro et al., 2019; Henkel et al., 2021).

The ample spectrum of diseases caused by C. trachomatis infection is reflected in its ability to infect and multiply within a wide range of different cell types, such as cervical epithelial cells, peripheral blood mononuclear cells (Dolat and Valdivia, 2019; Lausen et al., 2019). The typical model of chlamydial intracellular development has been mostly investigated in human cervical epithelial cells (HeLa) and murine fibroblasts (McCoy), for which C. trachomatis possesses the highest tropism (Belland et al., 2003; Guseva et al., 2007; Vromman et al., 2014; Petyaev et al., 2017; Sessa et al., 2017a; Filardo et al., 2019a; Liang and Mahony, 2019; Jøraholmen et al., 2020). C. trachomatis developmental cycle occurs entirely within a cell-derived membrane bound vesicle termed inclusion, where Chlamydiae alternate between the elementary body (EB), the extracellular and infectious form, and the reticulate body (RB), the metabolically active form, responsible for intracellular replication (AbdelRahman and Belland, 2005). The first stage of chlamydial developmental cycle consists in EBs adhesion to host cell membrane receptors, like glycosaminoglycans (Conant and Stephens, 2007). Then, chlamydial EBs enter the host cell by endocytosis, via a two-step process involving a reversible interaction mediated by heparin-sulphate proteoglycans followed by irreversible binding to host receptors (Conant and Stephens, 2007). Soon after attachment to the host cell, EBs are internalized and confined to the inclusion, where they differentiate to RBs; within 24 hours post-infection (h.p.i.), chlamydial RBs replicate by binary fission (Bastidas et al., 2013). As inclusion expands, approximately $24-48$ h.p.i., the majority of RBs begin to transition back to EBs in an asynchronous process (AbdelRahman and Belland, 2005). At the end of the developmental cycle, at about 48 h.p.i., the EBs are finally released from the host by cell lysis or extrusion (Yang et al., 2015; Zuck et al., 2016). Thereafter, a multitude of infectious EBs spread and infect neighboring cells, perpetuating the infectious process.

To date, the human cervical epithelial cell has been the most studied cellular infection model, focusing on chlamydial growth and on the peculiar features of the host-cell inflammatory and immune responses to the infection (Lad et al., 2005; Sessa et al., 2017b; Tang et al., 2021). Following chlamydial infection, the host cell response typically begins with the activation of a complex network of immune receptors (TLR2 and TR4) and their respective downstream signaling pathways (myeloid differentiation primary response $88, \mathrm{MyD} 88$, and nuclear factor kappa-light-chain-enhancer of activated B cells, NFkB) (O'Connell et al., 2006; Sellami et al., 2014). This results in the induction of proinflammatory cytokines, involved in either the elimination of C. trachomatis or tissue damage related to chronic inflammatory state, including interleukin (IL)-1 $\alpha$, IL-6, IL-8 and interferon (IFN)- $\boldsymbol{\gamma}$ (O'Connell et al., 2006; Rey-Ladino et al., 2014; Sellami et al., 2014). IFN- $\gamma$, in particular, has been identified as a major player in the clearance and protection against C. trachomatis infection, by modulating a plethora of host cell signalling pathways, like the activation of NF-kB and the inflammasome network (Rothfuchs et al., 2004; Webster et al., 2017).

Overall, the pathogenic mechanisms underlying $C$. trachomatis-mediated chronic complications have received the most research attention in women, whereas chlamydial survival strategies as well as the host defense pathways, involved in the onset and development of prostatitis, male infertility, and reactive arthritis, are now beginning to emerge. Therefore, herein we provide the up-to-date evidence on the interaction between C. trachomatis and human prostate epithelial, Sertoli and synovial cells.

\section{TRACHOMATIS INFECTION MODELS IN PROSTATITIS, MALE INFERTILITY AND REACTIVE ARTHRITIS}

\section{Human Prostate Epithelial Cells}

The first evidence to demonstrate $C$. trachomatis growth within primary human prostate epithelial cells came from Greenberg et al, in 1985 (Greenberg et al., 1985). Since then, few studies on chlamydial interaction with prostate epithelial cells were performed, describing both the ability of C. trachomatis to replicate in these cells and the specific host-cell inflammatory and immune pathways in response to the infection. Specifically, a study investigating the inflammatory profile of $C$. trachomatis infection in humane prostate epithelial cells (PNT2) and urethral epithelial cells (THUEC), demonstrated that prostate epithelial cells produced larger quantities of IL- 6 and IL- 8 than urethral epithelial cells, suggesting the increased levels of these cytokines as possible markers for $C$. trachomatis infection of the prostate (Al-Mously and Eley, 2007).

At a later time, a potential link between the inflammatory damage and C. trachomatis infection of the prostate was suggested, since a strong pro-inflammatory response, characterized by NFkB activation and TLR2/TLR4 upregulation, was observed, leading to increased inflammatory cytokine expression, like IL-6, IL-8, IL-1 $\beta$ and tumor necrosis factor (TNF) $\alpha$ (Sellami et al., 2014). Lastly, a recent study has described the efficient propagation of C. trachomatis in a malignant prostate epithelial cell line (CWR-R1) (proportion of infected cells 28.1\%) accompanied by enhanced transcription of IL- 6 and fibroblast growth factor (FGF)-2 genes, encoding two important pro-inflammatory cytokines involved in the progression of prostate cancer (Petyaev et al., 2019).

\section{Human Sertoli Cells}

In recent years, a study employing a murine model has postulated the direct infection of the seminiferous tubule epithelium, formed by Sertoli cells, as an interesting 
pathophysiological mechanism for C. trachomatis-mediated male infertility, leading to compromised spermatogenesis with reduced sperm count, motility and altered morphology of mature spermatozoa (Bryan et al., 2021).

On this basis, we have investigated, for the first time, the interaction between C. trachomatis and human primary Sertoli cells in vitro, demonstrating a distinct growth profile of $C$. trachomatis, with a very long eclipse period (after 36 h.p.i.), the appearance of infectious EBs beyond 48 h.p.i., the persistence of inclusions up to 96 h.p.i. and a low infection efficiency (Filardo et al., 2019b). This greatly differed from the chlamydial growth cycle as typically seen in cervical epithelial cells, where the transition of RBs to EBs happen after 22 h.p.i. and the release of infectious EBs from host cells is usually observed 36 to 48 h.p.i. (Guseva et al., 2007; Vromman et al., 2014; Skilton et al., 2018). Of great pathological importance, the development of $C$. trachomatis inclusions has also been demonstrated to visibly damage the host cell cytoskeleton, as shown by the reorganization of Vimentin-based intermediate filaments and $\alpha$-tubulin microtubules in thick fibres surrounding chlamydial inclusions (Filardo et al., 2019b). This, in turn, might alter the integrity of the blood-testis barrier, a structural compartment of the seminiferous tubules essential for germ cell development and maturation, impairing the spermatogenesis and contributing to male infertility (Mruk and Cheng, 2015). On this regard, the evidence that human spermatozoa were unaffected by biomolecules produced by chlamydial infected Sertoli cells, suggests that $C$. trachomatis is more likely to influence the early stages leading to the development of mature spermatozoa.

In addition to structural damage, C. trachomatis was also demonstrated to modulate the immune response in human Sertoli cells, characterized by the activation of TLR3 alongside the down-modulation of downstream signaling pathways, namely NFKB and interferon regulatory factor (IRF)3 (Di Pietro et al., 2020a). Consequently, IFNs type-I and type-II, IL$1 \alpha$ and IL- 6 were not produced, suggesting that C. trachomatis could evade the host immune-mediated killing, surviving in the cells and damaging the testicular tissue.

\section{Human Synovial Cells}

The interaction between $C$. trachomatis and human synovial cells was investigated in 1998 by Rödel et al., showing, for the first time, the ability of C. trachomatis to infect fibroblast-like cells derived from biopsies of the synovial membrane (Rödel et al., 1998a). Then, C. trachomatis infection of synovial cells was also demonstrated to elicit the production of several proinflammatory cytokines, such as IL- 6 and IFN- $\beta$ (Rödel et al., 1998b).

Since then, few studies have further researched this interaction, detailing some cellular mechanisms underlying the synovial cell immune response to chlamydial infection. In particular, increased production of IRF1 and interferonstimulated gene factor (ISGF)-3 $\gamma$ was observed in synovial cells infected with $C$. trachomatis, leading to the production of IFN $\beta$ as well as to the upregulation of Human Leukocyte Antigen (HLA)-1 gene expression (Rödel et al., 1999).
In recent years, the unique morphology, and the peculiar growth cycle of chlamydial inclusions in a primary human synovial cell have been described, and differed significantly from those in cervical epithelial cells. In particular, $C$. trachomatis was characterized by heterogeneous shape and size of inclusions and by a delayed developmental cycle, with late appearing infectious EBs (after 36 h.p.i.), as well as by a lower infection efficiency (Filardo et al., 2021).

The investigation of synovial cell immune response toward C. trachomatis evidenced a distinct profile characterized by the activation of TLR3 and TLR2 as well as of downstream signaling molecules, like ISG56 and Guanylate Binding Protein (GBP)1, interferon-inducible proteins involved in the cell-autonomous immunity against intracellular bacteria (MacMicking, 2012; Di Pietro et al., 2020b; Honkala et al., 2020). Nevertheless, the synovial cell response to $C$. trachomatis seemed ineffective in controlling the infection, suggesting its potential evasion of synovial cell inflammatory pathways (Filardo et al., 2021). Indeed, the increased expression of caspase-1 gene, in Chlamydia infected synovial cells, did not induce a parallel increase in the production of IL-6 as well as IL-1 $\beta$ and IL-18. A further interesting evidence is the observation that caspase activation played an important role in the intracellular growth of C. trachomatis in synovial cells, as demonstrated by decreased chlamydial replication after caspase inhibition (Filardo et al., 2021).

Differently, synovial cells were demonstrated to be able to control chlamydial infection when exposed to IFN $\gamma$, a wellknown pro-inflammatory cytokine involved in the clearance of C. trachomatis genital infection. In particular, IFN $\gamma$ was demonstrated to inhibit chlamydial growth decreasing caspase1 gene expression while, at the same time, inducing TLR2 and ISG56 gene expression and IL1 $\beta$, IL-18 and IL- 6 production, suggesting the key role of IFN $\gamma$ for the modulation of inflammatory and immune responses of synovial cells toward C. trachomatis (Di Pietro et al., 2020b).

\section{DISCUSSION}

Research to elucidate how C. trachomatis interact with specific cells of the prostate, male genital tract and joints is fundamental to understand how these interactions influence disease outcomes. To date, the available data provide insights regarding $C$. trachomatis growth within human prostate epithelial, Sertoli and synovial cells, and the related host-cell response pathways (Figure 1).

Human prostate epithelial cells showed a similar progression of chlamydial intracellular developmental cycle as that observed in cervical epithelial cells, alongside a comparable infection efficiency (Petyaev et al., 2019). By contrast, in Sertoli and synovial cells, the duration of the different C. trachomatis developmental stages, as well as the number of infectious EBs released from host cells, greatly differed as compared to those observed in cervical epithelial cells, routinely used for chlamydial research (Filardo et al., 2019b). The late appearances of infectious 




FIGURE 1 | Schematic representation of the immune and inflammatory pathways elicited by C. trachomatis infection of human prostate epithelial, Sertoli and synovial cells.

EBs toward the end of the developmental cycle suggests the presence of hostile cellular environment that may partially hinder chlamydial intracellular growth in these cells. In addition, the lower infection efficiency of C. trachomatis, previously described, in human Sertoli and synovial cells as compared to prostate epithelial cells, highlights that the prostate is particularly susceptible to $C$. trachomatis infection and, hence, could represent a trojan horse for the subsequent dissemination in the host, including the epididymis/testis or the joint.

Important differences were also observed in the immune and inflammatory host cell responses to C. trachomatis infection, with the activation of different molecular sensors, downstream signalling pathways and inflammatory signatures. Indeed, human prostate cells recognition of C. trachomatis by TLR2/ TLR4 induced a pro-inflammatory state, highlighted by increased levels of IL-1 $\beta$, IL-6, IL-8 and TNF $\alpha$ (Sellami et al., 2014; Petyaev et al., 2019). By contrast, TLR3-mediated sensing of chlamydial infection in human Sertoli cells did not elicit the activation of the related pathways, namely NFkB and IRF3, as well as the subsequent cytokine production. These results hint that in human prostate epithelial cells inflammation may play a key role in the pathogenesis of $C$. trachomatis-mediated tissue damage and prostate cancer progression. Instead, in Sertoli cells, C. trachomatis might induce direct cell-damage, as evidenced by the alteration of host-cell cytoskeleton, and, at the same time, remain within the cell for a long time, leading to a chronic infection. Indeed, in Sertoli cells, C. trachomatis appears to modulate the innate immune response, evading, hence, the host immune-mediate killing (Di Pietro et al., 2020a).

A further evasion strategy from host-cell defence pathways was also described in human synovial cells, as shown by $C$. trachomatis hijack of caspase-1 mediated inflammasome network, inhibiting the production of IL- 6 as well as Il-1 $\beta$ and IL-18, involved in pyroptosis, a cellular defence mechanism against infectious agents (Man et al., 2017; Filardo et al., 2021). 
Surprisingly, earlier reports demonstrated the induction of a proinflammatory state in human synovial cells infected by $C$. trachomatis, evidenced by increased IL-6 levels (Rödel et al., 1998b; Rödel et al., 1999), whereas our recent studies evidenced a significant increase in pro-inflammatory cytokine levels following the exposure to IFN $\gamma$ (Di Pietro et al., 2020b). These discordant results may be dependent on several factors, like, for example, the $C$. trachomatis serovar, since early reports used $C$. trachomatis serovar $\mathrm{E}$, whereas the serovar $\mathrm{D}$ was investigated in recent studies (Rödel et al., 1998b; Filardo et al., 2021). The serotype D and E have also been used in studies involving Sertoli and prostate epithelial cells (Greenberg et al., 1985; Al-Mously and Eley, 2007; Filardo et al., 2019b; Di Pietro et al., 2020a); the lymphogranuloma venereum serovar L2 was used in prostate malignant cells (Sellami et al., 2014; Petyaev et al., 2019).

Overall, the path ahead is still long, and, in the future, more complex approaches with $3 \mathrm{D}$ cell cultures and organoids will be helpful for shedding light on the etiopathogenesis of

\section{REFERENCES}

AbdelRahman, Y. M., and Belland, R. J. (2005). The Chlamydial Developmental Cycle: Figure 1. FEMS Microbiol. Rev. 29, 949-959. doi: 10.1016/ j.femsre.2005.03.002

Al-Mously, N., and Eley, A. (2007). Interaction of Chlamydia Trachomatis Serovar E With Male Genital Tract Epithelium Results in Secretion of Proinflammatory Cytokines. J. Med. Microbiol. 56, 1025-1032. doi: 10.1099/ jmm.0.47241-0

Badalyan, R. R., Fanarjyan, S. V., and Aghajanyan, I. G. (2003). Chlamydial and Ureaplasmal Infections in Patients With Nonbacterial Chronic Prostatitis. Andrologia 35, 263-265. doi: 10.1046/j.1439-0272.2003.00582.x

Bastidas, R. J., Elwell, C. A., Engel, J. N., and Valdivia, R. H. (2013). Chlamydial Intracellular Survival Strategies. Cold Spring Harbor Perspect. Med. 3, a010256a010256. doi: 10.1101/cshperspect.a010256

Belland, R. J., Zhong, G., Crane, D. D., Hogan, D., Sturdevant, D., Sharma, J., et al. (2003). Genomic Transcriptional Profiling of the Developmental Cycle of Chlamydia Trachomatis. Proc. Natl. Acad. Sci. 100, 8478-8483. doi: 10.1073/ pnas. 1331135100

Bryan, E. R., Barrero, R. A., Cheung, E., Tickner, J. A. D., Trim, L. K., Richard, D., et al. (2021). DNA Damage Contributes to Transcriptional and Immunological Dysregulation of Testicular Cells During Chlamydia Infection. Am. J. Reprod. Immunol. 86. doi: 10.1111/aji.13400

Conant, C. G., and Stephens, R. S. (2007). Chlamydia Attachment to Mammalian Cells Requires Protein Disulfide Isomerase. Cell. Microbiol. 9, 222-232. doi: $10.1111 / j .1462-5822.2006 .00783 . x$

Di Pietro, M., Filardo, S., Alfano, V., Pelloni, M., Splendiani, E., Po, A., et al. (2020a). Chlamydia Trachomatis Elicits TLR3 Expression But Disrupts the Inflammatory Signaling Down-Modulating Nfאb and IRF3 Transcription Factors in Human Sertoli Cells. J. Biol. Regul. Homeost. Agents 34, 977-986. doi: $10.23812 / 20-80-\mathrm{A}-29$

Di Pietro, M., Filardo, S., Frasca, F., Scagnolari, C., Manera, M., Sessa, V., et al. (2020b). Interferon- $\gamma$ Possesses Anti-Microbial and Immunomodulatory Activity on a Chlamydia Trachomatis Infection Model of Primary Human Synovial Fibroblasts. Microorganisms 8. doi: 10.3390/microorganisms8020235

Di Pietro, M., Filardo, S., Romano, S., and Sessa, R. (2019). Chlamydia Trachomatis and Chlamydia Pneumoniae Interaction With the Host: Latest Advances and Future Prospective. Microorganisms 7. doi: 10.3390/ microorganisms 7050140

Dolat, L., and Valdivia, R. H. (2019). A Renewed Tool Kit to Explore Chlamydia Pathogenesis: From Molecular Genetics to New Infection Models. F1000Research 8. doi: 10.12688/f1000research.18832.1
C. trachomatis-mediated prostatitis, male infertility, and reactive arthritis, since many cell signalling pathways, that might have been elicited in response to chlamydial infection, are still unexplored.

\section{AUTHOR CONTRIBUTIONS}

MDP and RS conceived the manuscript. SF and FD performed literature review. SF, MDP, and RS wrote the original manuscript. SF, MDP, and RS revised the manuscript. SF and FD designed the Figure. All authors approved the submitted version.

\section{FUNDING}

This research was funded by the University of Rome "Sapienza" to Prof. Rosa Sessa (grant RP11916B6AEB0D37).

Filardo, S., Di Pietro, M., Frasca, F., Diaco, F., Scordio, M., Antonelli, G., et al. (2021). Potential Ifn $\gamma$ Modulation of Inflammasome Pathway in Chlamydia Trachomatis Infected Synovial Cells. Life 11, 1359. doi: 10.3390/life11121359

Filardo, S., Di Pietro, M., Tranquilli, G., and Sessa, R. (2019a). Biofilm in Genital Ecosystem: A Potential Risk Factor for Chlamydia Trachomatis Infection. Can. J. Infect. Dis. Med. Microbiol. 2019. doi: 10.1155/2019/1672109

Filardo, S., Skilton, R. J., O’Neill, C. E., Di Pietro, M., Sessa, R., and Clarke, I. N. (2019b). Growth Kinetics of Chlamydia Trachomatis in Primary Human Sertoli Cells. Sci. Rep. 9. doi: 10.1038/s41598-019-42396-3

Greenberg, S. B., Harris, D., Giles, P., Martin, R. R., and Wallace, R. J. (1985). Inhibition of Chlamydia Trachomatis Growth in McCoy, HeLa, and Human Prostate Cells by Zinc. Antimicrob Agents Chemother 27, 953-957. doi: 10.1128/AAC.27.6.953

Guseva, N. V., Dessus-Babus, S., Moore, C. G., Whittimore, J. D., and Wyrick, P. B. (2007). Differences in Chlamydia Trachomatis Serovar E Growth Rate in Polarized Endometrial and Endocervical Epithelial Cells Grown in ThreeDimensional Culture. Infect Immun. 75, 553-564. doi: 10.1128/IAI.01517-06

Henkel, R., Offor, U., and Fisher, D. (2021). The Role of Infections and Leukocytes in Male Infertility. Andrologia 53. doi: 10.1111/and.13743

Honkala, A. T., Tailor, D., and Malhotra, S. V. (2020). Guanylate-Binding Protein 1: An Emerging Target in Inflammation and Cancer. Front. Immunol. 10. doi: 10.3389/fimmu.2019.03139

Jøraholmen, M. W., Johannessen, M., Gravningen, K., Puolakkainen, M., Acharya, G., Basnet, P., et al. (2020). Liposomes-In-Hydrogel Delivery System Enhances the Potential of Resveratrol in Combating Vaginal Chlamydia Infection. Pharmaceutics 12. doi: 10.3390/pharmaceutics 12121203

Khan, F. U., Ihsan, A. U., Khan, H. U., Jana, R., Wazir, J., Khongorzul, P., et al. (2017). Comprehensive Overview of Prostatitis. Biomed Pharmacother 94, 1064-1076. doi: 10.1016/j.biopha.2017.08.016

Krieger, J. N., Lee, S. W. H., Jeon, J., Cheah, P. Y., Liong, M. L., and Riley, D. E. (2008). Epidemiology of Prostatitis. Int. J. Antimicrob. Agents 31, 85-90. doi: 10.1016/j.ijantimicag.2007.08.028

Lad, S. P., Fukuda, E. Y., Li, J., de la Maza, L. M., and Li, E. (2005). Up-Regulation of the JAK/STAT1 Signal Pathway During Chlamydia Trachomatis Infection. J. Immunol. 174, 7186-7193. doi: 10.4049/jimmunol.174.11.7186

Lausen, M., Christiansen, G., Bouet Guldbæk Poulsen, T., and Birkelund, S. (2019). Immunobiology of Monocytes and Macrophages During Chlamydia Trachomatis Infection. Microbes Infect 21, 73-84. doi: 10.1016/ j.micinf.2018.10.007

Liang, S., and Mahony, J. B. (2019). Enumeration of Viable Chlamydia From Infected Animals Using Immunofluorescent Microscopy. Methods Mol. Biol. (Clifton N.J.) 2042, 237-244. doi: 10.1007/978-1-4939-9694-0_16 
MacMicking, J. D. (2012). Interferon-Inducible Effector Mechanisms in CellAutonomous Immunity. Nat. Rev. Immunol. 12, 367-382. doi: 10.1038/nri3210

Man, S. M., Karki, R., and Kanneganti, T.-D. (2017). Molecular Mechanisms and Functions of Pyroptosis, Inflammatory Caspases and Inflammasomes in Infectious Diseases. Immunol. Rev. 277, 61-75. doi: 10.1111/imr.12534

Mruk, D. D., and Cheng, C. Y. (2015). The Mammalian Blood-Testis Barrier: Its Biology and Regulation. Endocr Rev. 36, 564-591. doi: 10.1210/er.2014-1101

O'Connell, C. M., and Ferone, M. E. (2016). Chlamydia Trachomatis Genital Infections. Microb Cell (Graz Austria) 3. doi: 10.15698/mic2016.09.525

O’Connell, C. M., Ionova, I. A., Quayle, A. J., Visintin, A., and Ingalls, R. R. (2006). Localization of TLR2 and MyD88 to Chlamydia Trachomatis Inclusions. J. Biol. Chem. 281, 1652-1659. doi: 10.1074/jbc.M510182200

Ostaszewska, I., Zdrodowska-Stefanow, B., Badyda, J., Pucilo, K., Trybula, J., and Bulhak, V. (1998). Chlamydia Trachomatis : Probable Cause of Prostatitis. Int. J. STD AIDS 9, 350-353. doi: 10.1258/0956462981922395

Ouzounova-Raykova, V., Ouzounova, I., and Mitov, I. G. (2010). May Chlamydia Trachomatis be an Aetiological Agent of Chronic Prostatic Infection? Andrologia 42, 176-181. doi: 10.1111/j.1439-0272.2009.00973.x

Petyaev, I. M., Zigangirova, N. A., Morgunova, E. Y., Kyle, N. H., Fedina, E. D., and Bashmakov, Y. K. (2017). Resveratrol Inhibits Propagation of Chlamydia Trachomatis in McCoy Cells. BioMed. Res. Int. 2017, 1-7. doi: 10.1155/2017/ 4064071

Petyaev, I. M., Zigangirova, N. A., Morgunova, E. Y., Kyle, N. H., Fedina, E. D., and Bashmakov, Y. K. (2019). Chlamydia Trachomatis Growth and Cytokine mRNA Response in a Prostate Cancer Cell Line. Adv. Urol. 2019, 1-8. doi: 10.1155/2019/6287057

Rey-Ladino, J., Ross, A. G., and Cripps, A. W. (2014). Immunity, Immunopathology, and Human Vaccine Development Against Sexually Transmitted Chlamydia Trachomatis. Hum. Vaccines Immunotherapeutics 10, 2664-2673. doi: 10.4161/hv.29683

Rödel, J., Groh, A., Vogelsang, H., Lehmann, M., Hartmann, M., and Straube, E. (1998a). Beta Interferon Is Produced by Chlamydia Trachomatis -Infected Fibroblast-Like Synoviocytes and Inhibits Gamma Interferon-Induced HLADR Expression. Infect Immun. 66, 4491-4495. doi: 10.1128/IAI.66.9.44914495.1998

Rödel, J., Groh, A., Hartmann, M., Schmidt, K.-H., Lehmann, M., Lungershausen, W., et al. (1999). Expression of Interferon Regulatory Factors and Indoleamine 2,3-Dioxygenase in Chlamydia Trachomatis- Infected Synovial Fibroblasts. Med. Microbiol. Immunol. 187, 205-212. doi: 10.1007/s004300050094

Rödel, J., Straube, E., Lungershausen, W., Hartmann, M., and Groh, A. (1998b). Secretion of Cytokines by Human Synoviocytes During In Vitro Infection With Chlamydia Trachomatis. J. Rheumatol. 25, 2161-2168.

Rothfuchs, A. G., Trumstedt, C., Wigzell, H., and Rottenberg, M. E. (2004). Intracellular Bacterial Infection-Induced IFN- $\gamma$ Is Critically But Not Solely Dependent on TollLike Receptor 4-Myeloid Differentiation Factor 88-IFN- $\alpha \beta$-STAT1 Signaling. J. Immunol. 172, 6345-6353. doi: 10.4049/jimmunol.172.10.6345

Rowley, J., vander Hoorn, S., Korenromp, E., Low, N., Unemo, M., Abu-Raddad, L. J., et al. (2019). Chlamydia, Gonorrhoea, Trichomoniasis and Syphilis: Global Prevalence and Incidence Estimate. Bull. World Health Organ. 97. doi: 10.2471/ BLT.18.228486

Sellami, H., Said-Sadier, N., Znazen, A., Gdoura, R., Ojcius, D. M., and Hammami, A. (2014). Chlamydia Trachomatis Infection Increases the Expression of Inflammatory Tumorigenic Cytokines and Chemokines as Well as Components of the Toll-Like Receptor and NF- $\mathrm{b}$ P Pathways in Human Prostate Epithelial Cells. Mol. Cell. Probes 28, 147-154. doi: 10.1016/ j.mcp.2014.01.006
Sessa, R., Di Pietro, M., Filardo, S., Bressan, A., Mastromarino, P., Biasucci, A. V., et al. (2017a). Lactobacilli-Lactoferrin Interplay in Chlamydia Trachomatis Infection. Pathog. Dis. 75. doi: 10.1093/femspd/ftx054

Sessa, R., Di Pietro, M., Filardo, S., Bressan, A., Rosa, L., Cutone, A., et al. (2017b). Effect of Bovine Lactoferrin on Chlamydia Trachomatis Infection and Inflammation. Biochem. Cell Biol. 95, 34-40. doi: 10.1139/bcb-2016-0049

Skilton, R. J., Wang, Y., O’Neill, C., Filardo, S., Marsh, P., Bénard, A., et al. (2018). The Chlamydia Muridarum Plasmid Revisited: New Insights Into Growth Kinetics [Version 1; Referees: 2 Approved, 1 Approved With Reservations]. Wellcome Open Res. 3. doi: 10.12688/wellcomeopenres.13905.1

Tang, C., Liu, C., Maffei, B., Niragire, B., Cohen, H., Kane, A., et al. (2021). Primary Ectocervical Epithelial Cells Display Lower Permissivity to Chlamydia Trachomatis Than HeLa Cells and a Globally Higher Pro-Inflammatory Profile. Sci. Rep. 11, 5848. doi: 10.1038/s41598-021-85123-7

Thoma, M., Fledderjohann, J., Cox, C., and Kantum Adageba, R. (2021). "Biological and Social Aspects of Human Infertility: A Global Perspective," in Oxford Research Encyclopedia of Global Public Health (Oxford, UK: Oxford University Press). doi: 10.1093/acrefore/9780190632366.013.184

Trinchieri, A., Abdelrahman, K. M., Bhatti, K. H., Bello, J. O., Das, K., Gatsev, O., et al. (2021). Spectrum of Causative Pathogens and Resistance Rates to Antibacterial Agents in Bacterial Prostatitis. Diagnostics 11, 1333. doi: 10.3390/diagnostics11081333

Vromman, F., Laverrière, M., Perrinet, S., Dufour, A., and Subtil, A. (2014). Quantitative Monitoring of the Chlamydia Trachomatis Developmental Cycle Using GFP-Expressing Bacteria, Microscopy and Flow Cytometry. PloS One 9, e99197. doi: 10.1371/journal.pone.0099197

Webster, S. J., Brode, S., Ellis, L., Fitzmaurice, T. J., Elder, M. J., Gekara, N. O., et al. (2017). Detection of a Microbial Metabolite by STING Regulates Inflammasome Activation in Response to Chlamydia Trachomatis Infection. PloS Pathog. 13, e1006383. doi: 10.1371/journal.ppat.1006383

Yang, C., Starr, T., Song, L., Carlson, J. H., Sturdevant, G. L., Beare, P. A., et al. (2015). Chlamydial Lytic Exit From Host Cells Is Plasmid Regulated. mBio 6. doi: $10.1128 / \mathrm{mBio} .01648-15$

Zeidler, H., and Hudson, A. (2016). Coinfection of Chlamydiae and Other Bacteria in Reactive Arthritis and Spondyloarthritis: Need for Future Research. Microorganisms 4, 30. doi: 10.3390/microorganisms4030030

Zuck, M., Sherrid, A., Suchland, R., Ellis, T., and Hybiske, K. (2016). Conservation of Extrusion as an Exit Mechanism for Chlamydia. Pathog. Dis. 74, ftw093. doi: $10.1093 /$ femspd/ftw093

Conflict of Interest: The authors declare that the research was conducted in the absence of any commercial or financial relationships that could be construed as a potential conflict of interest.

Publisher's Note: All claims expressed in this article are solely those of the authors and do not necessarily represent those of their affiliated organizations, or those of the publisher, the editors and the reviewers. Any product that may be evaluated in this article, or claim that may be made by its manufacturer, is not guaranteed or endorsed by the publisher.

Copyright (C) 2022 Filardo, Di Pietro, Diaco and Sessa. This is an open-access article distributed under the terms of the Creative Commons Attribution License (CC BY). The use, distribution or reproduction in other forums is permitted, provided the original author(s) and the copyright owner(s) are credited and that the original publication in this journal is cited, in accordance with accepted academic practice. No use, distribution or reproduction is permitted which does not comply with these terms. 